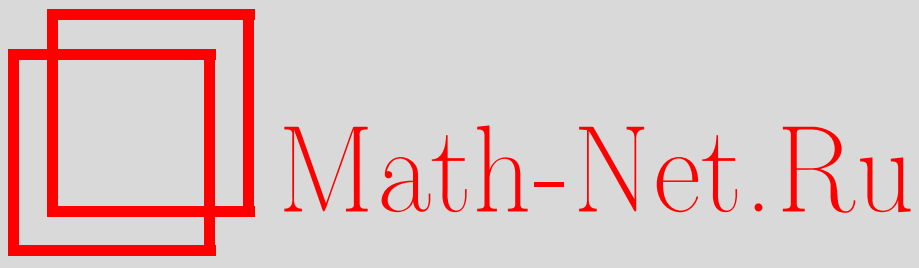

А. Н. Кочубей, Фундаментальные решения псевдодифференциальных уравнений, связанных с р-адическими квадратичными формами, Изв. РАН. Сер. матем., 1998, том 62, выпуск 6, 103-124

DOI: https://doi.org/10.4213/im222

Использование Общероссийского математического портала Math-Net.Ru подразумевает, что вы прочитали и согласны с пользовательским соглашением

http://www.mathnet.ru/rus/agreement

Параметры загрузки:

IP : 54.197 .130 .99

26 апреля 2023 г., 18:07:38 
УДК 517.95

\author{
А.Н. Кочубей
}

\title{
Фундаментальные решения псевдодифференциальных уравнений, связанных с $p$-адическими квадратичными формами
}

\begin{abstract}
Строятся и исследуются фундаментальные решения, соответствующие некоторым классам псевдодифференциальных операторов с символами вида $|h(\xi)|_{p}^{\alpha}$, $\alpha>0$, где $h(\xi), \xi=\left(\xi_{1}, \ldots, \xi_{n}\right),-$ невырожденная $p$-адическая квадратичная форма.

Библиография: 28 наименований.
\end{abstract}

\section{§1. Введение}

Появление в квантовой механике и квантовой теории поля $p$-адических моделей (см. [1]-[3] и указанную там литературу) привело к значительной активизации исследований в области $p$-адического анализа, в том числе в теории обобщенных функций, в изучении различных классов операторов в функциональных пространствах над $p$-адическим полем $Q_{p}$ и над $Q_{p}^{n}=Q_{p} \times \cdots \times Q_{p}(n$ раз $)$. Ниже будут рассматриваться операторы в пространствах комплекснозначных функций на $Q_{p}^{n}$; случаю $Q_{p}$-значных функций посвящена монография [2].

Поскольку $Q_{p}$ вполне несвязно, комплекснозначную функцию $u(x), x \in Q_{p}$, нельзя дифференцировать. Поэтому естественно рассмотреть псевдодифференциальные операторы. Простейший пример - оператор дробного дифференцирования, введенный В. С. Владимировым [4]:

$$
\left(D^{\alpha} z\right)(x)=F_{\xi \rightarrow x}^{-1}\left[|\xi|_{p}^{\alpha} F_{y \rightarrow \xi} z\right], \quad \alpha>0
$$

где $F$ - преобразование $Ф$ урье,

$$
\begin{aligned}
& F_{y \rightarrow \xi} z=\int_{Q_{p}} \chi_{p}(y \xi) z(y) d y \\
& F_{\xi \rightarrow x}^{-1} \zeta=\int_{Q_{p}} \chi_{p}(-x \xi) \zeta(\xi) d \xi
\end{aligned}
$$

$\chi_{p}$ - канонический аддитивньй характер на $Q_{p}, d x$ - мера Хаара на аддитивной группе $Q_{p}$, нормированная условием

$$
\int_{|x|_{p} \leqslant 1} d x=1
$$

(C) А.Н. Кочувей 1998 
Выражение (1.1) имеет смысл для функции $z$, принадлежащей пространству основных функций $\mathscr{D}\left(Q_{p}\right)$, состоящему из локально постоянных функций с компактными носителями (см. [1]): если $z \in \mathscr{D}\left(Q_{p}\right)$, то $F z \in \mathscr{D}\left(Q_{p}\right)$ и $D^{\alpha} z \in L_{2}\left(Q_{p}\right)$. $\mathrm{C}$ другой стороны, $D^{\alpha}$ может быть представлен в виде гиперсингулярного интегрального оператора:

$$
\left(D^{\alpha} z\right)(x)=\frac{1-p^{\alpha}}{1-p^{-\alpha-1}} \int_{Q_{p}}|y|_{p}^{-\alpha-1}[z(x-y)-z(x)] d y
$$

$z \in \mathscr{D}\left(Q_{p}\right)$. Правая часть (1.2) имеет смысл для более широкого класса функций, например для всех локально постоянных функций $z$ таких, что $|z(x)| \leqslant C\left(|x|_{p}^{\nu}+1\right)$, $0 \leqslant \nu<\alpha$.

Фундаментальное решение уравнения

$$
D^{\alpha} z=f
$$

найдено в [4]:

$$
E(x)= \begin{cases}\frac{1-p^{-\alpha}}{1-p^{\alpha-1}}|x|_{p}^{\alpha-1}, & \text { если } \alpha \neq 1, \\ \frac{1-p}{p \log p} \log |x|_{p}, & \text { если } \alpha=1 .\end{cases}
$$

Если $f \in \mathscr{D}\left(Q_{p}\right)$, то $z=E * f$ локально постоянна, $|z(x)| \leqslant C\left(|x|_{p}^{\alpha-1}+1\right)$, и функция $z(x)$ удовлетворяет уравнению (1.3), где $D^{\alpha}$ понимается в смысле $(1.2)$.

Отметим, что непосредственному определению обобщенного решения $z \in$ $\mathscr{D}^{\prime}\left(Q_{p}\right)$ уравнения (1.1) препятствует то обстоятельство, что для функции $\varphi \in \mathscr{D}\left(Q_{p}\right)$ функция $D^{\alpha} \varphi$ может не принадлежать $\mathscr{D}\left(Q_{p}\right)$ (поскольку функция $\xi \mapsto|\xi|_{p}$ не является локально постоянной). В многомерных задачах ситуация еще сложнее. Например, известно [1], что уравнение $D_{t}^{1} z-D_{x}^{2} z=f(x, t)$, $x, t \in Q_{p}$, не имеет фундаментального решения из $\mathscr{D}^{\prime}\left(Q_{p}^{2}\right)$.

В настояшей работе рассматриваются многомерные псевдодифференциальные операторы вида

$$
(A z)(x)=F_{\xi \rightarrow x}^{-1}\left[|h(\xi)|_{p}^{\alpha} F_{y \rightarrow \xi} z\right], \quad x \in Q_{p}^{n}
$$

где $h(\xi), \xi=\left(\xi_{1}, \ldots, \xi_{n}\right) \in Q_{p}^{n},-$ квадратичная форма с $p$-адическими коэффициентами, $\alpha>0$,

$$
\begin{aligned}
& F_{y \rightarrow \xi} z=\int_{Q_{p}^{n}} \chi\left(y_{1} \xi_{1}+\cdots+y_{n} \xi_{n}\right) z\left(y_{1}, \ldots, y_{n}\right) d y_{1} \ldots d y_{n} \\
& F_{\xi \rightarrow x}^{-1} \zeta=\int_{Q_{p}^{n}} \chi\left(-x_{1} \chi_{1}-\cdots-x_{n} \xi_{n}\right) \zeta\left(\xi_{1}, \ldots, \xi_{n}\right) d \xi_{1} \ldots d \xi_{n}
\end{aligned}
$$

Будем предполагать, что $p \neq 2$. 
Очевидная аналогия подсказывает три класса операторов (1.5). Эллиптическими операторами естественно называть те, для которых $h(\xi) \neq 0$ при $\xi \neq 0$ (см. также [5]). В отличие от классической ситуации, эллиптические операторы над $Q_{p}^{n}$ существуют только при $n \leqslant 4$ и допускают полную классификацию (см. [6], [7]). Для каждого из них строится гиперсингулярное интегральное представление и фундаментальное решение. Применяемая для этой цели методика состоит в сведении задачи к случаю одномерных псевдодифференциальных операторов над локальными полями (расширениями $Q_{p}$; в случае $n=4$ удобнее использовать некоммутативное тело кватернионов над $Q_{p}$ ). Этот подход уже применялся ранее [8] при исследовании функции Грина оператора $A$, рассматриваемого как самосопряженный оператор в $L_{2}\left(Q_{p}\right)$; последняя задача связана с намеченной в [9] перспективой построения $p$-адического аналога евклидовой квантовой теории поля.

Операторы (1.5) с $h(\xi)=\xi_{1}^{2}-g\left(\xi_{2}, \ldots, \xi_{n}\right), \quad g\left(\xi_{2}, \ldots, \xi_{n}\right) \neq 0$, если $\left(\xi_{2}, \ldots, \xi_{n}\right) \neq 0$, можно назвать гиперболическими. Простейший случай $n=2$ легко сводится к одномерному. Случай, когда $n>2$, сложнее, поскольку решение может быть обобщенным, а действие оператора $A$ в $\mathscr{D}^{\prime}\left(Q_{p}^{n}\right)$ не определено. Обозначим

$$
\begin{gathered}
C=\left\{\xi \in Q_{p}^{n}: \xi_{1}^{2}=g\left(\xi_{2}, \ldots, \xi_{n}\right)\right\}, \\
\mathscr{D}_{C}\left(Q_{p}^{n}\right)=\left\{\varphi \in \mathscr{D}\left(Q_{p}^{n}\right): \widetilde{\varphi}(\xi)=0, \quad \xi \in C\right\}
\end{gathered}
$$

(здесь и ниже вместо $F \varphi$ часто используется обозначение $\widetilde{\varphi}$ ). Тогда $A: \mathscr{D}_{C}^{\prime}\left(Q_{p}^{n}\right) \rightarrow$ $\mathscr{D}_{C}^{\prime}\left(Q_{p}^{n}\right)$, и нетрудно построить фундаментальное решение $E \in \mathscr{D}_{C}^{\prime}\left(Q_{p}^{n}\right)$, даюшее решение $u=E * f$ уравнения $A u=f$ с $f \in \mathscr{D}_{C}\left(Q_{p}^{n}\right)$ (см. [10] по поводу дальнейшего развития этого подхода, применимого не только к гиперболическим операторам). Оказывается, что в гиперболическом случае для любого $\alpha$, кроме двух исключительных значений, фундаментальное решение $E$ можно продолжить до обобщенной функции из $\mathscr{D}^{\prime}\left(Q_{p}^{n}\right)$.

"Параболический" случай, когда $h(\xi)=\xi_{1}-g\left(\xi_{2}, \ldots, \xi_{n}\right)$ и $g$-невырожденная квадратичная форма, уже изучен в [11]. В этом случае свойства фундаментальных решений подобны случаю классического нестационарного уравнения Шрёдингера. В самом деле, оказалось [11], что уравнению $A u=0$ с таким $A$ удовлетворяет свободная волновая функция $p$-адической квантовой механики, развитой в [1], [3], [9]. Подлинно "параболический” характер имеют уравнения с вешественной переменной и $p$-адическими пространственными переменными, изученные в [12].

\section{§2. Эллиптические уравнения}

2.1. Одномерные псевдодифференциальные операторы над локальным полем. Пусть $K$ - полное несвязное недискретное локально компактное поле, снабженное ультраметрическим нормализованным абсолютным значением $\|\cdot\|$ (по поводу основных сведений о локальных полях см. [13]-[15]). Обозначим

$$
O=\{x \in K:\|x\| \leqslant 1\}, \quad P=\{x \in K:\|x\|<1\} .
$$


Кольцо $O$ назьвается кольцом цельх поля $K, P$ - простой идеал в $O$. Поле вычетов $O / P$ состоит из $q<\infty$ элементов. Идеал $P$ содержит такой элемент $\beta$, что $P=\beta O,\|\beta\|=q^{-1}$. Абсолютная величина $\|x\|$ принимает значения $q^{N}$, $N \in \mathbb{Z}$. Каждый элемент $x \in K$ можно представить в виде $x=\beta^{-N} u_{x}$, где $\|x\|=q^{N},\left\|u_{x}\right\|=1$.

Нормируем меру Хаара $d x$ на аддитивной группе $K$, полагая, что мера $O$ равна 1. Преобразование Фурье на $K$ определяется так же, как для $K=Q_{p}$; следует интегрировать по $K$ и использовать аддитивный характер $\chi$ рангануль $(\chi(x)=1$, если $x \in O$, и $\chi(a) \neq 1$ для некоторого $a \in K$ c $\|a\|=q)$ вместо $\chi_{p}$. Иногда будет употребляться запись $\widetilde{\psi}$ вместо $F \psi$. Эти же обозначения будут использоваться для преобразования Фурье обобщенных функций (по поводу обобщенных функций над локальными полями см. [14], [16]).

Рассмотрим псевдодифференциальный оператор

$$
\mathfrak{A}=F^{-1} a F
$$

где символ $a$ имеет вид

$$
a(\xi)=\|\xi\|^{\nu} \varphi\left(u_{\xi}\right), \quad \xi \in K
$$

$\nu>0, \varphi-$ непрерьвная строго положительная функция на группе единиц $U=$ $O \backslash P=\{u \in K:\|u\|=1\}$. Пусть $\mathscr{D}(K)$ - пространство основных функций, состоящее из локально постоянных функций с компактными носителями (иногда оно обозначается $\mathscr{S}(K)$, чтобы подчеркнуть его инвариантность относительно преобразования Фурье). Тогда $\mathfrak{A}: \mathscr{D}(K) \rightarrow L_{2}(K)$.

Далее будет предполагаться, что $\varphi$ удовлетворяет следующему условию локального постоянства: сушествует такое натуральное число $m$, что из неравенства $\|v\| \leqslant q^{-m}$ следует равенство

$$
\varphi(u+v)=\varphi(u) \quad \text { для всех } u \in U \text {. }
$$

Из (2.3) вытекает (см. [8]), что $\varphi$ - конечная линейная комбинация характеров мультипликативной групшы $U$ :

$$
\varphi(u)=\sum_{j=0}^{m} \sum_{l=1}^{L_{j}} c_{j l} \theta_{j l}(u), \quad u \in U
$$

где $c_{j l} \in \mathbb{C}, \theta_{j l}$ - характер ранга $j$ (см. [14], [16]); количество таких характеров, обозначаемое $L_{j}$, равно единище для $j=0, q-2$ для $j=1$ и $q^{j-2}(q-1)^{2}$ для $j \geqslant 2[16]$.

В [16] доказано, что

$$
(\mathfrak{A} z)(x)=\int_{K}\|y\|^{-\nu-1} \Omega\left(u_{y}\right)[z(x-y)-z(x)] d y
$$


где

$$
\Omega(u)=\sum_{j=0}^{m} \sum_{l=1}^{L_{j}} c_{j l}\left[\Gamma\left(q^{\nu}, \overline{\theta_{j l}}\right)\right]^{-1} \overline{\theta_{j l}(u)}, \quad u \in U
$$

и Г - гамма-функция, связанная с полем $K$ (см. [14], [16]; мы следуем обозначениям из [14]).

Правая часть гиперсингулярного интегрального представления (2.4) оператора $\mathfrak{A}$ имеет смысл для всех локально постоянных функций $z(x)$, удовлетворяющих условию

$$
|z(x)| \leqslant C\left(1+|x|_{p}^{\mu}\right), \quad 0 \leqslant \mu<\nu .
$$

Назовем локально постоянную функцию $z(x)$ обобщенныц решением уравнения

$$
\mathfrak{A} z=f, \quad f \in \mathscr{D}(K),
$$

если $z$ удовлетворяет (2.5) и (2.6), где $\mathfrak{A}$ понимается в смысле (2.4).

Построим фундаментальное решение $E$ для уравнения (2.6). В данном случае $E$ будет обычной функцией, непрерывной при $x \neq 0$ и такой, что $z=E * f-$ обобшенное решение (2.6).

Поскольку $\varphi$ строго положительна, $\varphi^{-1}$ обладает свойством, аналогичным $(2.3)$. Следовательно,

$$
[\varphi(u)]^{-1}=\sum_{j=0}^{m} \sum_{l=1}^{L_{j}} c_{j l}^{\prime} \theta_{j l}(u), \quad u \in U
$$

для некоторых $c_{j l}^{\prime} \in \mathbb{C}$. Из $(2.1)$ и $(2.2)$ видно, что $(F E)(\xi)$ формально совпадает c $\|\xi\|^{-\nu}\left[\varphi\left(u_{\xi}\right)\right]^{-1}$. В следующем предположении дана регуляризация обратного преобразования Фурье.

ПРЕДЛОЖЕНИЕ 2.1. Функиия

$$
E(x)=\|x\|^{\nu-1} \sum_{j=0}^{m} \sum_{l=1}^{L_{j}} c_{j l}^{\prime}\left[\Gamma\left(q^{-\nu}, \overline{\theta_{j l}}\right)\right]^{-1} \overline{\theta_{j l}\left(u_{x}\right)}, \quad \nu \neq 1
$$

или

$$
E(x)=-c_{01} \frac{q-1}{q \log q} \log \|x\|+\sum_{j=1}^{m} \sum_{l=1}^{L_{j}} c_{j l}^{\prime}\left[\Gamma\left(q^{-1}, \overline{\theta_{j l}}\right)\right]^{-1} \overline{\theta_{j l}\left(u_{x}\right)}, \quad \nu=1
$$

является фундаментальным решением для уравнения (2.6). 
ДоКАЗАТЕЛЬСТво. Ясно, что $z=E * f$ - локально постоянная функция,

$$
|z(x)| \leqslant \begin{cases}C\left(\|x\|^{\max (\nu-1,0)}+1\right), & \text { если } \nu \neq 1, \\ C \log (2+\|x\|), & \text { если } \nu=1 .\end{cases}
$$

Здесь и ниже буквой $C$ обозначаются различные положительные постоянные. Далее будет рассматриваться случай $\nu=1$; случай $\nu \neq 1$ проще и может быть исследован аналогично.

Замечая из (2.4), что функция $\mathfrak{A} z$ локально постоянна, мы можем интерпретировать ее как обобщенную функцию из $\mathscr{D}^{\prime}(K)$ и рассмотреть ее преобразование Фурье. Если $\psi \in \mathscr{D}(K)$, то

$$
\langle F \mathfrak{A} z, \psi\rangle=\langle\mathfrak{A} z, \widetilde{\psi}\rangle=\int_{K}\|y\|^{-2} \Omega\left(u_{y}\right) d y \int_{K}[z(x-y)-z(x)] \widetilde{\psi}(x) d x .
$$

Обозначив $\psi_{y}(t)=\chi(y t) \psi(t)$, получим

$$
\int_{K}[z(x-y)-z(x)] \widetilde{\psi}(x) d x=\left\langle\tilde{z}, \psi_{y}-\psi\right\rangle=\left\langle\widetilde{E}, \tilde{f}\left(\psi_{y}-\psi\right)\right\rangle .
$$

Обобщенную функцию $\widetilde{E}$ можно вычислить, пользуясь формулами из [1], [16]:

$$
\widetilde{E}(\xi)=c_{01}\left[\mathscr{P}\|\xi\|^{-1}+q^{-1} \delta(\xi)\right]+\sum_{j=1}^{m} \sum_{l=1}^{L_{j}} c_{j l}\|\xi\|^{-1} \theta_{j l}\left(u_{\xi}\right),
$$

где

$$
\left\langle\mathscr{P}\|\xi\|^{-1}, \psi\right\rangle=\int_{\|\xi\| \leqslant 1}[\psi(\xi)-\psi(0)]\|\xi\|^{-1} d \xi+\int_{\|\xi\|>1}\|\xi\|^{-1} \psi(\xi) d \xi .
$$

Поскольку функция $\tilde{f}\left(\psi_{y}-\psi\right)$ равна нулю вблизи нуля, находим

$$
\left\langle\widetilde{E}, \tilde{f}\left(\psi_{y}-\psi\right)\right\rangle=\int_{K}\|\xi\|^{-1}\left[\varphi\left(u_{\xi}\right)\right]^{-1} \tilde{f}(\xi)[\chi(y \xi)-1] \psi(\xi) d \xi .
$$

Вводя обозначения

$$
\begin{aligned}
& R_{l}(\xi)=\left\{\begin{array}{lll}
\|\xi\|^{-1}\left[\varphi\left(u_{\xi}\right)\right]^{-1} \tilde{f}(\xi) \psi(\xi) & \text { при } & \|\xi\|>q^{l}, \\
0 & \text { при } & \|\xi\| \leqslant q^{l},
\end{array}\right. \\
& I_{l}(y)=\int_{\|\xi\| \leqslant q^{l}}\|\xi\|^{-1}\left[\varphi\left(u_{\xi}\right)\right]^{-1} \tilde{f}(\xi)[\chi(y \xi)-1] \psi(\xi) d \xi,
\end{aligned}
$$

получаем

$$
\begin{aligned}
\langle F \mathfrak{A} z, \psi\rangle= & \int_{K}\|y\|^{-2} \Omega\left(u_{y}\right)\left[\widetilde{R}_{l}(y)-\widetilde{R}_{l}(0)\right] d y \\
& +\int_{K}\|y\|^{-2} \Omega\left(u_{y}\right) I_{l}(y) d y
\end{aligned}
$$


для любого $l \in \mathbb{Z}$.

Обобщенная функция $\|y\|^{-2} \Omega\left(u_{y}\right)$ (регуляризация функции с неинтегрируемой особенностью; см. [1], [16]) является преобразованием Фурье функции $\|\xi\| \varphi\left(u_{\xi}\right)$. Отсюда

$$
\begin{aligned}
\int_{K}\|y\|^{-2} \Omega\left(u_{y}\right)\left[\widetilde{R}_{l}(y)-\widetilde{R}_{l}(0)\right] d y & =\int_{K}\|\xi\| \varphi\left(u_{\xi}\right) R_{l}(\xi) d \xi \\
& =\int_{\|\xi\|>q^{l}} \tilde{f}(\xi) \psi(\xi) d \xi \rightarrow\langle\tilde{f}, \psi\rangle, \quad l \rightarrow-\infty
\end{aligned}
$$

Здесь было использовано то, что $R_{l} \in \mathscr{D}(K)$.

Рассмотрим второй интеграл в (2.7). Для любого $y \in K I_{l}(y) \rightarrow 0$ при $l \rightarrow-\infty$. Если $l \leqslant 0$, то $I_{l}(y)=0$ при $\|y\| \leqslant 1$,

$$
\begin{aligned}
\left|I_{l}(y)\right| & \leqslant C \int_{\|y\|-1 \leqslant\|\xi\| \leqslant 1}\|\xi\|^{-1} d \xi \\
& =C \sum_{j=-\log _{q}\|y\|}^{0} \int_{\|\xi\|=q^{j}}\|\xi\|^{-1} d \xi \leqslant C\left[\log _{q}\|y\|+1\right]
\end{aligned}
$$

при $\|y\|>1$. Из теоремы Лебега следует, что второе слагаемое в (2.7) стремится к нулю при $l \rightarrow \infty$.

Итак,

$$
\langle F \mathfrak{A} z, \psi\rangle=\langle\tilde{f}, \psi\rangle
$$

откуда $\mathfrak{A} z=f$. Предложение доказано.

Для оператора $D_{K}^{\nu}$, соответствующего символу $a(\xi)=\|\xi\|^{\nu}$, получается

$$
E(x)= \begin{cases}\frac{1-q^{-\nu}}{1-q^{\nu-1}}\|x\|^{\nu-1}, & \text { если } \nu \neq 1, \\ \frac{1-q}{q \log q} \log \|x\|, & \text { если } \nu=1,\end{cases}
$$

так же, как в случае $K=Q_{p}$ (см. (1.4)). Этот результат остается в силе и тогда, когда $K$ - недискретное вполне несвязное локально компактное некоммутативное тело. Все понятия и конструкции теории локальных полей, использованные для доказательства формулы (2.8), применимы и в этой ситуации (см. [17]-[19]). Формула $(2.8)$ для тела кватернионов над $Q_{p}$ будет использоваться ниже.

2.2. Координатное представление. Пусть $K$ имеет характеристику нуль. Тогда $K-$ расширение поля $Q_{p}$ конечной степени $n$. Предположим, что $n \geqslant 2$. Характер ранга нуль адлитивной группы $K$ можно задать формулой

$$
\chi(x)=\chi_{p}\left(\operatorname{Tr}\left(\beta^{-d} x\right)\right),
$$

где $\operatorname{Tr}: K \rightarrow Q_{p}$ - след в $K$ над $Q_{p}, d$ - показатель дифференты (см. [15]). 
Рассмотрим билинейную форму

$$
\rho(x, y)=\operatorname{Tr}\left(x y \pi^{-d}\right), \quad x, y \in K
$$

со значениями в $Q_{p}$. Поскольку $\rho$ симметрична и не вырождена, в поле $K$ существует $\rho$-ортогональный базис $e_{1}, \ldots, e_{n}$ над $Q_{p}$ (см. [20]):

$$
\rho\left(e_{i}, e_{j}\right)=0, \quad i \neq j ; \quad \rho\left(e_{i}, e_{i}\right)=\mu_{i} \neq 0, \quad i=1, \ldots, n
$$

Если $x=x_{1} e_{1}+\cdots+x_{n} e_{n}, y=y_{1} e_{1}+\cdots+y_{n} e_{n}, x_{i}, y_{i} \in Q_{p}$, то

$$
\chi(x y)=\prod_{i=1}^{n} \chi_{p}\left(\mu_{i} x_{i} y_{i}\right)
$$

Нормированную аддитивную меру Хаара на $K$ можно представить в виде

$$
d x=c_{K} d x_{1} \ldots d x_{n}
$$

где $d x_{j}$ - нормированная аддитивная мера Хаара на $Q_{p}$,

$$
c_{K}^{-1}=\int_{\left\|x_{1} e_{1}+\cdots+x_{n} e_{n}\right\| \leqslant 1} d x_{1} \ldots d x_{n}
$$

Пусть $\mathfrak{A}$ - псевдодифференциальный оператор (2.1). Рассмотрим псевдодифференциальный оператор $B$ над $Q_{p}$, определенный соотношением

$$
(B g)\left(x_{1}, \ldots, x_{n}\right)=(\mathfrak{A} f)\left(x_{1} e_{1}+\cdots+x_{n} e_{n}\right)
$$

где $g \in \mathscr{D}\left(Q_{p}^{n}\right) ; f \in \mathscr{D}(K)$ такова, что

$$
g\left(x_{1}, \ldots, x_{n}\right)=f\left(x_{1} e_{1}+\cdots+x_{n} e_{n}\right), \quad x_{j} \in Q_{p}
$$

Легко видеть, что $B$ - псевдодифференциальный оператор с символом

$$
b\left(\xi_{1}, \ldots, \xi_{n}\right)=c_{K}^{2}\left(\prod_{j=1}^{n}\left|\mu_{j}\right|_{p}^{-1}\right) a\left(\sum_{k=1}^{n} \mu_{k}^{-1} \xi_{k} e_{k}\right) .
$$

Базис $\left\{e_{1}, \ldots, e_{n}\right\}$ называется базисом координатного представления для оператора $\mathfrak{A}$. Ниже (как и в [8]) такой базис будет часто использоваться следуюшим образом. Учитывая то, что эллиптический оператор $A$ над $Q_{p}^{n}$ имеет вид (1.5), находим расширение $K$, базис координатного представления, символ $a(\xi), \xi \in K$, вида (2.2) и матрицу $T$ порядка $n$ над $Q_{p}$ такие, что

$$
\left|h\left(\xi_{1}, \ldots, \xi_{n}\right)\right|_{p}^{\alpha}=b\left(T\left(\xi_{1}, \ldots, \xi_{n}\right)\right)
$$


(мы не различаем вектор-строки и вектор-столбцы), где $b$ имеет вид (2.12). Теперь $A=W_{T}^{-1} B W_{T},\left(W_{T} g\right)(x)=g\left(T^{\prime} x\right), x \in Q_{p}^{n}$. Из гиперсингулярного интегрального представления (2.4) следует представление

$$
\begin{aligned}
(A g)\left(x_{1}, \ldots, x_{n}\right)= & \int_{K}\|y\|^{-\nu-1} \Omega\left(u_{y}\right)\left[g\left(x_{1}-\left(T^{\prime} Y\right)_{1}, \ldots, x_{n}-\left(T^{\prime} Y\right)_{n}\right)\right. \\
& \left.-g\left(x_{1}, \ldots, x_{n}\right)\right] d y
\end{aligned}
$$

где $Y=\left(y_{1}, \ldots, y_{n}\right) \in Q_{p}^{n}$ определяется из разложения $y=y_{1} e_{1}+\cdots+y_{n} e_{n}$, $y \in K$. Связь между $\alpha$ и $\nu$, а также способ выбора $K$ и $a$ будут конкретно указаны для различных $n$ и тех или иных классов форм $h$.

Выражение (2.13) имеет смысл для локально постоянных функций $g\left(x_{1}, \ldots, x_{n}\right)$, растуших на бесконечности не быстрее, чем $\max _{1 \leqslant j \leqslant n}\left|x_{j}\right|_{p}^{\nu-\varepsilon}, \varepsilon>0$. Если $E(x)-$ фундаментальное решение для оператора $\mathfrak{A}$, построенное в предложении 2.1 , то фундаментальное решение $G\left(x_{1}, \ldots, x_{n}\right)$ для оператора $A$ (понимаемое точно так же, как в п. 2.1) дается формулой

$$
G\left(x_{1}, \ldots, x_{n}\right)=|\operatorname{det} T|_{p}^{-1} E\left(\left(T^{\prime-1} X\right)_{1} e_{1}+\cdots+\left(T^{\prime-1} X\right)_{n} e_{n}\right),
$$

где $X=\left(x_{1}, \ldots, x_{n}\right)$. Эта формула проверяется непосредственным вычислением.

Соотношения между операторами $A$ и $\mathfrak{A}$, в том числе формула (2.14), остаются в силе, когда $K$ - (некоммутативное) тело. Пусть, в частности, $K$ - центральная алгебра с делением над $Q_{p}, \operatorname{dim} K=m^{2}$ (см. [15], [18]). В (2.9) и (2.10) нужно только заменить $d$ на $m-1$, а $\operatorname{Tr}$ - на приведенный след $t$. Гиперсингулярные представления (2.4) для общих символов (2.2) возможны только для коммутативных $K$. Однако все рассуждения, относяшиеся к оператору $D_{K}^{\alpha}$, справедливы и в случае некоммутативного тела.

\section{3. Фундаментальные решения для эллиптических операторов.}

Teopema 2.1. Ecлu

$$
h\left(\xi_{1}, \ldots, \xi_{n}\right) \neq 0 \quad \text { npu } \quad\left|\xi_{1}\right|_{p}+\cdots+\left|\xi_{n}\right|_{p} \neq 0
$$

то для оператора А существует фундаментальное решение $G\left(x_{1}, \ldots, x_{n}\right)$, непрерывное при $\left(x_{1}, \ldots, x_{n}\right) \neq 0$ и имеющее не более чем степенной рост на бесконечности. Если $f \in \mathscr{D}\left(Q_{p}^{n}\right)$, то функиия $z=G * f$ удовлетворяет уравнению $A z=f$, где $A$ понимается как гиперсингулярный интегральный оператор (2.13).

Доказательство, приведенное ниже, содержит явные конструкции $G$ для всех возможных квадратичных форм, удовлетворяющих условию эллиптичности (2.15). При этом указывается также явньй вид соответствующих гиперсингулярных интегральных представлений (2.13). 
2.4. Бинарные формы. Пусть $h\left(\xi_{1}, \xi_{2}\right)$ удовлетворяет (2.15). Тогда с точностью до линейного изоморфизма

$$
h\left(\xi_{1}, \xi_{2}\right)=\xi_{1}^{2}-\tau \xi_{2}^{2},
$$

где $\tau \in Q_{p}$ не является квадратом в $Q_{p}[6],[7]$. Чтобы получить все неэквивалентные формы, нужно взять полньй набор представителей $\tau$ классов из $Q_{p}^{*} / Q_{p}^{* 2}$ (здесь $Q_{p}^{*}$-мультипликативная группа поля $Q_{p}$, a $Q_{p}^{* 2}$ - подгруппа всех квадратов):

$$
\tau=\varepsilon, \quad|\varepsilon|_{p}=1 ; \quad \tau=p ; \quad \tau=\varepsilon p .
$$

В каждом из трех случаев положим $K=Q_{p}(\sqrt{\tau})$. Абсолютная величина элемента $x=x_{1}+\sqrt{\tau} x_{2} \in K$ равна

$$
\|x\|=\left|x_{1}^{2}-\tau x_{2}^{2}\right|_{p}
$$

(отметим, что здесь используется нормализованная абсолютная величина [13]). Оператор умножения на $x=x_{1}+\sqrt{\tau} x_{2}$ представляется матрицей $\left(\begin{array}{cc}x_{1} & x_{2} \\ x_{2} \tau & x_{1}\end{array}\right)$ относительно базиса $\{1, \sqrt{\tau}\}$. Следовательно, $\operatorname{Tr} x=2 x_{1}$.

Рассмотрим отдельно каждый из возможных случаев.

Пусть $\tau=\varepsilon$. Тогда $K$ не разветвлено над $Q_{p}[21]$, откуда $q=p^{2}, d=0$. В качестве базиса координатного представления можно взять $e_{1}=1, e_{2}=\sqrt{\varepsilon}$. Нетрудно подсчитать [8], что $c=1, \mu_{1}=2, \mu_{2}=2 \varepsilon,\left|\mu_{1}\right|_{p}=\left|\mu_{2}\right|_{p}=1$. Полагая $\nu=\alpha, \mathfrak{A}=D_{K}^{\alpha}, T=\left(\begin{array}{cc}2 & 0 \\ 0 & 2 \varepsilon\end{array}\right)$, получаем гиперсингулярное интегральное представление

$$
\begin{aligned}
(A g)\left(x_{1}, x_{2}\right)= & \frac{1-p^{2 \alpha}}{1-p^{-2 \alpha-2}} \int_{Q_{p}^{2}}\left|y_{1}^{2}-\varepsilon y_{2}^{2}\right|_{p}^{-\alpha-1} \\
& \times\left[g\left(x_{1}-2 y_{1}, x_{2}-2 \varepsilon y_{2}\right)-g\left(x_{1}, x_{2}\right)\right] d y_{1} d y_{2} .
\end{aligned}
$$

Фундаментальное решение имеет вид

$$
G\left(x_{1}, x_{2}\right)=E\left(\frac{x_{1}}{2}+\sqrt{\varepsilon} \frac{x_{2}}{2 \varepsilon}\right), \quad x_{1}, x_{2} \in Q_{p},
$$

где

$$
E(x)= \begin{cases}\frac{1-p^{-2 \alpha}}{1-p^{2(\alpha-1)}}\|x\|^{\alpha-1}, & \text { если } \alpha \neq 1, \\ \frac{1-p^{2}}{2 p^{2} \log p} \log \|x\|, & \text { если } \alpha=1,\end{cases}
$$

для всех $x \in K, x \neq 0$.

Пусть $\tau=p$. Тогда $K$-вполне разветвленное расширение $Q_{p}[21], q=p, d=1$. Можно положить $\beta=\sqrt{p}$ и взять

$$
e_{1}=1-\sqrt{p}, \quad e_{2}=1+\sqrt{p}
$$


в качестве базиса координатного представления. Тогда [8] $c=1, \mu_{1}=-4, \mu_{2}=4$, $\left|\mu_{1}\right|_{p}=\left|\mu_{2}\right|_{p}=1$. Как и вьше, $\nu=\alpha, \mathfrak{A}=D_{K}^{\alpha}$. В данном случае

$$
\begin{gathered}
T=\frac{1}{2}\left(\begin{array}{cc}
1 & 1 \\
-1 & 1
\end{array}\right), \\
(A g)\left(x_{1}, x_{2}\right)=\frac{1-p^{\alpha}}{1-p^{-\alpha-1}} \int_{Q_{p}^{2}}\left|\left(y_{1}+y_{2}\right)^{2}-p\left(y_{2}-y_{1}\right)^{2}\right|_{p}^{-\alpha-1} \\
\times\left[g\left(x_{1}-\frac{y_{1}}{2}+\frac{y_{2}}{2}, x_{2}-\frac{y_{1}}{2}-\frac{y_{2}}{2}\right)-g\left(x_{1}, x_{2}\right)\right] d y_{1} d y_{2} .
\end{gathered}
$$

Выражение для фундаментального решения имеет вид

$$
G\left(x_{1}, x_{2}\right)=E\left(\left(x_{1}+x_{2}\right)(1-\sqrt{p})+\left(x_{2}-x_{1}\right)(1+\sqrt{p})\right)=E\left(2 x_{2}-2 x_{1} \sqrt{p}\right)
$$

где

$x \in K, x \neq 0$.

$$
E(x)= \begin{cases}\frac{1-p^{-\alpha}}{1-p^{\alpha-1}}\|x\|^{\alpha-1}, & \text { если } \alpha \neq 1, \\ \frac{1-p}{p \log p} \log \|x\|, & \text { если } \alpha=1,\end{cases}
$$

Третий случай $(\tau=\varepsilon p)$ практически не отличается от второго.

2.5. Тернарные формы. Если квадратичная форма $h\left(\xi_{1}, \xi_{2}, \xi_{3}\right)$ удовлетворяет (2.15), то с точностью до линейного изоморфизма [6]

$$
h\left(\xi_{1}, \xi_{2}, \xi_{3}\right)=p \varepsilon_{1} \xi_{1}^{2}+\varepsilon_{2} \xi_{2}^{2}+\varepsilon_{3} \xi_{3}^{2},
$$

где $\left|\varepsilon_{1}\right|_{p}=\left|\varepsilon_{2}\right|_{p}=\left|\varepsilon_{3}\right|_{p}=1, \varepsilon_{2} \xi_{2}^{2}+\varepsilon_{3} \xi_{3}^{2} \neq 0$, если $\left|\xi_{2}\right|_{p}+\left|\xi_{3}\right|_{p} \neq 0$. Поэтому достаточно рассмотреть формы вида (2.16).

Пусть $K$ - неразветвленное расширение $Q_{p}$ степени 3 . Его поле вычетов $\mathbb{F}_{q}=$ $O / P$, состояшее из $q=p^{3}$ элементов, является расширением простого конечного поля $\mathbb{F}_{p}=\mathbb{Z}_{p} / p \mathbb{Z}_{p}\left(\mathbb{Z}_{p}\right.$ и $p \mathbb{Z}_{p}$ - соответственно кольцо целых и простой идеал поля $Q_{p}$ ). Пусть $\lambda$ - примитивный элемент поля $\mathbb{F}_{q}$ (по поводу конечных полей см. [22]). Тогда элементы $1, \lambda, \lambda^{2}$ образуют базис в $\mathbb{F}_{q}$ над $\mathbb{F}_{p}$. Пусть $\left\{f_{1}, f_{2}, f_{3}\right\}-$ канонический базис в $K$ над $Q_{p}$, соответствующий базису $\left\{1, \lambda, \lambda^{2}\right\}$; это означает [8], что $f_{1}, f_{2}$ и $f_{3}$ - произвольные представители классов вычетов $1, \lambda$ и $\lambda^{2}$ соответственно (конечно, такая конструкция возможна только для неразветвленного расширения; см. [6]). Для абсолютной величины в $K$ имеется простое выражение в терминах коэффициентов разложения по каноническому базису (см. [23] или [8]): если $x \in K, x=\eta_{1} f_{1}+\eta_{2} f_{2}+\eta_{3} f_{3}$, то $\|x\|=\left[\max \left\{\left|\eta_{1}\right|_{p},\left|\eta_{2}\right|_{p},\left|\eta_{3}\right|_{p}\right\}\right]^{3}$.

Интерпретация оператора $A$, связанного с формой (2.16), как координатного представления псевдодифференциального оператора над $K$ найдена в [16]. Рассмотрим следуюшую функцию $\varphi$ на группе единиц $U$ поля $K$. Пусть $u \in U$, $u=\eta_{1} f_{1}+\eta_{2} f_{2}+\eta_{3} f_{3}$. Тогда $\max _{1 \leqslant j \leqslant 3}\left|\eta_{j}\right|_{p}=1$. Положим

$$
\varphi(u)= \begin{cases}p^{-\alpha}, & \text { если }\left|\eta_{1}\right|_{p}=1, \quad\left|\eta_{2}\right|_{p}<1, \quad\left|\eta_{3}\right|_{p}<1, \\ 1 & \text { в противном случае. }\end{cases}
$$


Оператор $\mathfrak{A}$ - это по определению псевдодифференциальный оператор над $K$ с символом

$$
a(\xi)=\|\xi\|^{2 \alpha / 3} \varphi\left(u_{\xi}\right), \quad \xi \in K .
$$

Если $\left\{e_{1}, e_{2}, e_{3}\right\}$ - базис координатного представления, то матрища $T$, фигурирующая в соотношениях между $\mathfrak{A}$ и $A$, равна $M \Theta^{\prime}$, где $M=\operatorname{diag}\left(\mu_{1}, \mu_{2}, \mu_{3}\right)$, а матрица $\Theta=\left(\theta_{i j}\right)$ преобразует базисы:

$$
f_{i}=\sum_{j=1}^{3} \theta_{i j} e_{j}, \quad i=1,2,3 .
$$

Разлагая $\varphi$ по характерам $\theta_{j l}$ группы $U$ и вычисляя соответствуюшие $\Gamma$-функции (см. п. 2.1), приходим к формуле

$$
\Omega(u)=\frac{1-p^{2 \alpha}}{1-p^{-2 \alpha-1}}\left(1-\frac{p^{-\alpha}-1}{p^{2}+p+1}\right)+\left(p^{-\alpha}-1\right) p^{2 \alpha}\left[S+\left(p^{2}+p+1\right)^{-1}\right],
$$

где

$$
S=\left\{\begin{array}{lll}
p-1, & \text { если } & \operatorname{tr}\left(\lambda^{N}\right)=0, \\
-1, & \text { если } & \operatorname{tr}\left(\lambda^{N}\right) \neq 0,
\end{array}\right.
$$

а $N$ определяется из представления $u=f_{2}^{N} y,\|1-y\|<1,0 \leqslant N \leqslant q-2 ; \operatorname{tr}-$ след из $\mathbb{F}_{q}$ в $\mathbb{F}_{p}$.

Отметим, что вычисление $\Omega(u)$ в доказательстве теоремы 3 из статьи [8] содержит неточность, приводящую к выражению, отличному от (2.17), хотя общий ход рассуждений в [8] верен; следует только учесть различия в выражениях для $\Gamma$-функций характеров $\theta_{j l}$ при $j=0$ и $j \geqslant 1$ [14]. Отметим также, что в [8] требовалось только доказать неравенство $\Omega(u)<0$, так что сама теорема 3 из [8] верна.

Практически те же вычисления вместе с предложением 2.1 приводят к формуле для фундаментального решения $E(x)$, соответствуюшего оператору $\mathfrak{A}$. Если $\alpha \neq$ $3 / 2$, то

$$
E(x)=\|x\|^{2 \alpha / 3-1} \rho\left(u_{x}\right), \quad x \in K,
$$

где для $u_{x}=f_{2}^{N} y,\|1-y\|<1$,

$$
\rho\left(u_{x}\right)=\left(1-\frac{1-p^{\alpha}}{p^{2}+p+1}\right) \frac{1-p^{-2 \alpha}}{1-p^{2 \alpha-3}}+\frac{\left(p^{\alpha}-1\right) p^{-2 \alpha+3}}{p^{2}+p+1},
$$

если $\operatorname{tr}\left(\lambda_{N}\right)=0$, и

$$
\rho\left(u_{x}\right)=\left(1-\frac{1-p^{\alpha}}{p^{2}+p+1}\right) \frac{1-p^{-2 \alpha}}{1-p^{2 \alpha-3}}-\frac{\left(p^{\alpha}-1\right)(p+1) p^{-2 \alpha+1}}{p^{2}+p+1},
$$

если $\operatorname{tr}\left(\lambda_{N}\right) \neq 0$. Если $\alpha=3 / 2$, то

$$
\begin{gathered}
E(x)=\frac{(p+2)(1-p)}{3 p^{2} \log p} \log \|x\|+\frac{(p-1)}{p^{3}\left(p^{2}+p+1\right)} \rho_{1}\left(u_{x}\right), \\
\rho_{1}\left(u_{x}\right)=\left\{\begin{array}{lll}
p^{3} & \text { при } & \operatorname{tr}\left(\lambda_{N}\right)=0, \\
-p(p+1) & \text { при } & \operatorname{tr}\left(\lambda_{N}\right) \neq 0 .
\end{array}\right.
\end{gathered}
$$


Как и ранее, фундаментальное решение $G$ для оператора $A$ дается формулой (2.14). Однако на этот раз выражение для $G$ не вполне явное, так как нет явного вида матрицы $T$. Ниже предлагается еше один подход, приводящий (при одном, возможно, несушественном, дополнительном предположении) к явному виду $G$.

Нам потребуются некоторые понятия, относяшиеся к базисам в расширениях конечных полей. Точнее, будут рассматриваться базисы в $\mathbb{F}_{q}, q=p^{3}$, над $\mathbb{F}_{p}$. Базис $\left\{\lambda_{1}, \lambda_{2}, \lambda_{3}\right\}$ называется автодуальныцм, если

$$
\operatorname{tr}\left(\lambda_{i} \lambda_{j}\right)=\left\{\begin{array}{lll}
1 & \text { при } i=j \\
0 & \text { при } i \neq j
\end{array}\right.
$$

он называется нормальнымм, если $\lambda_{2}=\lambda_{1}^{p}, \lambda_{3}=\lambda_{1}^{p^{2}}$, и примитивным, если $\lambda_{1}$, $\lambda_{2}, \lambda_{3}$ - примитивные элементы поля $\mathbb{F}_{q}$.

Для любого $p$ поле $\mathbb{F}_{q}$ обладает нормальным автодуальным базисом [24] и нормальным примитивным базисом [25]. Базис в $\mathbb{F}_{q}$, являюшийся одновременно автодуальным, нормальным и примитивным, сушествует, например, если $p=3$ или $p=5$. Это вытекает из явного вида полиномов [26], корни которых задают автодуальный нормальный базис, и приведенной в [22] таблицы примитивных полиномов. Представляется вероятным, что автодуальный нормальный примитивньй базис существует при любом $p$.

Предположим теперь, что $\mathbb{F}_{q}$ обладает автодуальным нормальным примитивным базисом $\left\{\lambda_{1}, \lambda_{2}, \lambda_{3}\right\}$ над $\mathbb{F}_{p}$. Как и ранее, через $K$ будет обозначаться неразветвленное расширение $Q_{p}$ степени 3 .

ПРЕДЛОЖЕНИЕ 2.2. Поле $К$ обладает базисом координатного представления $\left\{e_{1}, e_{2}, e_{3}\right\}$, являющ,имся каноническим базисом; классы $\hat{e}_{1}, \hat{e}_{2}, \hat{e}_{3}$ әлементов е в $\mathbb{F}_{q}=O / P$ образуют базис в $\mathbb{F}_{q}$ над $\mathbb{F}_{p}$.

ДокАЗАТЕЛЬСТво. Из автодуальности базиса $\left\{\lambda_{1}, \lambda_{2}, \lambda_{3}\right\}$ и теоремы 90 Гильберта следует сушествование таких ненулевых элементов $\zeta_{12}, \zeta_{13}, \zeta_{23} \in \mathbb{F}_{q}$, что

$$
\lambda_{1} \lambda_{2}=\zeta_{12}^{p}-\zeta_{12}, \quad \lambda_{1} \lambda_{3}=\zeta_{13}^{p}-\zeta_{13}, \quad \lambda_{2} \lambda_{3}=\zeta_{23}^{p}-\zeta_{23} .
$$

Группа единиц $U$ содержит такой элемент $u_{0}$, что $u_{0}^{q-1}=1$ и множество $M=$ $\left\{u_{0}, u_{0}^{2}, \ldots, u_{0}^{q-1}\right\}$ - полный набор представителей классов вычетов из $O / P$. Пусть $s_{12}, s_{13}, s_{23} \in M-$ представители соответственно классов $\zeta_{12}, \zeta_{13}, \zeta_{23}$. Тогда $s_{i j}^{p}=\sigma_{K}\left(s_{i j}\right)$, где $\sigma_{K}-$ автоморфизм $\Phi$ робениуса [15].

Пусть $e_{1}^{0}, e_{2}^{0}, e_{3}^{0} \in U$ - произвольные представители классов $\lambda_{1}, \lambda_{2}, \lambda_{3}$. Из тождеств (2.18) следует, что

$$
e_{i}^{0} e_{j}^{0}-\left(s_{i j}^{p}-s_{i j}\right) \equiv 0 \quad(\bmod P), \quad 1 \leqslant i<j \leqslant 3 .
$$

Рассмотрим систему уравнений

$$
e_{i} e_{j}-\left(s_{i j}^{p}-s_{i j}\right)=0, \quad 1 \leqslant i<j \leqslant 3,
$$


относительно $e_{1}, e_{2}, e_{3} \in K$. Определитель соответствующей матрицы Якоби равен $-2 e_{1} e_{2} e_{3}$; при $e_{j}=e_{j}^{0}$ он является обратимым элементом кольца $O$ (так как $p \neq 2$ ). Из леммы Гензеля для системы уравнений (см. [17]) вытекает сушествование решения $\left\{e_{j}\right\}$ системы $(2.20)$ с $e_{j}-e_{j}^{0} \in P, j=1,2,3$. Поскольку $s_{i j}^{p}=\sigma_{K}\left(s_{i j}\right)$, имеем

$$
\operatorname{Tr}\left(e_{1} e_{2}\right)=\operatorname{Tr}\left(e_{1} e_{3}\right)=\operatorname{Tr}\left(e_{2} e_{3}\right)=0 .
$$

С другой стороны, $\hat{e}_{j}=\lambda_{j}, j=1,2,3$. Предложение доказано.

Из построения базиса $\left\{e_{1}, e_{2}, e_{3}\right\}$ и того факта, что $K$ не разветвлено над $Q_{p}$, получаем

$$
\left|\operatorname{Tr}\left(e_{j}^{2}\right)\right|_{p}=1, \quad j=1,2,3 .
$$

Построим теперь оператор $\mathfrak{A}$ так же, как это делалось выше, но взяв базис $\left\{e_{1}, e_{2}, e_{3}\right\}$ вместо $\left\{f_{1}, f_{2}, f_{3}\right\}$. В этом случае $T$ - единичная матрища, так что

$$
G\left(x_{1}, x_{2}, x_{3}\right)=E\left(x_{1} e_{1}+x_{2} e_{2}+x_{3} e_{3}\right) .
$$

Поскольку $\lambda_{1}$ - примитивный элемент $\mathbb{F}_{q}$, каждый элемент $u \in U$ единственным образом представим в виде

$$
u=e_{1}^{N} y, \quad 0 \leqslant N \leqslant q-2, \quad\|1-y\|<1 .
$$

Занумеруем характеры $\theta_{1 l}$ так, чтобы

$$
\theta_{1 l}(u)=\exp \left(\frac{2 \pi i l N}{q-1}\right), \quad 1 \leqslant l \leqslant q-2 .
$$

Повторяя рассуждения из [8], найдем выражения для коэффициентов $c_{j l}, c_{j l}^{\prime}$ (используются обозначения из п. 2.1). Имеем $m=1$,

$$
\begin{gathered}
c_{01}=1+\frac{p^{-\alpha}-1}{p^{2}+p+1} \\
c_{1 l}=\frac{p^{-\alpha}-1}{p^{2}+p+1} \exp \left(-\frac{2 \pi i \omega}{p^{2}+p+1}\right),
\end{gathered}
$$

если $l=\omega(p-1), \omega=1, \ldots, p(p+1)$, и $c_{1 l}=0$ для остальных значений $l \leqslant q-2$;

$$
\begin{gathered}
c_{01}^{\prime}=1+\frac{p^{\alpha}-1}{p^{2}+p+1} \\
c_{1 l}^{\prime}=\frac{p^{\alpha}-1}{p^{2}+p+1} \exp \left(-\frac{2 \pi i \omega}{p^{2}+p+1}\right),
\end{gathered}
$$

если $l=\omega(p-1), \omega=1, \ldots, p(p+1)$, и $c_{1 l}^{\prime}=0$ для остальных значений $l \leqslant q-2$.

Вычислим для данной ситуации функции $\Omega$ и $E$, исходя из формул п. 2.1 c $\nu=2 \alpha / 3$. 
Пусть $\alpha \neq 3 / 2$. Пользуясь тождествами [14], [16]

$$
\begin{aligned}
\Gamma\left(q^{-2 \alpha / 3}, 1\right) & =\frac{1-q^{2 \alpha / 3-1}}{1-q^{-2 \alpha / 3}}, \\
{\left[\Gamma\left(q^{-2 \alpha / 3}, \theta_{1, \nu(p-1)}\right)\right]^{-1} } & =\frac{\Gamma\left(q^{-1+2 \alpha / 3}, \theta_{1, \nu(p-1)}\right)}{}
\end{aligned}
$$

и очевидным тождеством

$$
\overline{\theta_{1, \nu(p-1)}}=\theta_{1,\left(p^{2}+p+1-\nu\right)(p-1)}, \quad 1 \leqslant \nu \leqslant p^{2}+p,
$$

после замены индекса суммирования получаем

$$
\begin{aligned}
E(x)= & \|x\|^{2 \alpha / 3-1}\left[\left(1+\frac{p^{\alpha}-1}{p^{2}+p+1}\right) \frac{1-p^{-2 \alpha}}{1-p^{2 \alpha-3}}+\frac{p^{\alpha}-1}{p^{2}+p+1}\right. \\
& \left.\times \sum_{\nu=1}^{p(p+1)} \overline{\Gamma\left(q^{-1+2 \alpha / 3}, \theta_{1, \nu(p-1)}\right)} \exp \left(\frac{2 \pi i \nu}{p^{2}+p+1}\right) \theta_{1, \nu(p-1)}\left(u_{x}\right)\right] .
\end{aligned}
$$

Известно [14], [16], что

$$
\Gamma\left(q^{-1+2 \alpha / 3}, \theta_{1, l}\right)=q^{1-2 \alpha / 3} \int_{\|y\|=1} \chi\left(p^{-1} y\right) \theta_{1, l}(y) d y .
$$

Этот интеграл равен сумме интегралов по множествам

$$
\left\{y=e_{1}^{m} z, \quad\|1-z\|<1\right\}, \quad 0 \leqslant m \leqslant q-2 .
$$

Мера каждого из них равна $\left(1-q^{-1}\right)(q-1)^{-1}=q^{-1}$ в силу инвариантности меры. Поэтому

$$
\Gamma\left(q^{-1+2 \alpha / 3}, \theta_{1, l}\right)=q^{-2 \alpha / 3} \sum_{m=0}^{q-2} \chi\left(p^{-1} e_{1}^{m}\right) \exp \left(\frac{2 \pi i l m}{q-1}\right) .
$$

Записьвая $u_{x}$ в форме $(2.22)$, находим

$$
\begin{aligned}
E(x)= & \|x\|^{2 \alpha / 3-1}\left[\left(1+\frac{p^{\alpha}-1}{p^{2}+p+1}\right) \frac{1-p^{-2 \alpha}}{1-p^{2 \alpha-3}}\right. \\
& +\frac{p^{-2 \alpha}\left(p^{\alpha}-1\right)}{p^{2}+p+1} \sum_{m=0}^{q-2} \sum_{\nu=1}^{p(p+1)} \chi\left(-p^{-1} e_{1}^{m}\right) \\
& \left.\times \exp \left(\frac{2 \pi i \nu(N-m+1)}{p^{2}+p+1}\right)\right] .
\end{aligned}
$$


Известные свойства тригонометрических сумм [22] приводят к тождествам

$$
\begin{aligned}
& \sum_{\nu=0}^{p(p+1)} \exp \left(\frac{2 \pi i \nu(N-m+1)}{p^{2}+p+1}\right) \\
& = \begin{cases}p^{2}+p+1, & \text { если } N-m+1=j\left(p^{2}+p+1\right), \quad j \in \mathbb{Z}, \\
0 & \text { в противном случае },\end{cases} \\
& \sum_{m=0}^{q-2} \chi\left(-p^{-1} e_{1}^{m}\right)=-1 .
\end{aligned}
$$

Отсюда

$$
\begin{aligned}
& \sum_{m=0}^{q-2} \sum_{\nu=1}^{p(p+1)} \chi\left(-p^{-1} e_{1}^{m}\right) \exp \left(\frac{2 \pi i \nu(N-m+1)}{p^{2}+p+1}\right) \\
& \quad=\left(p^{2}+p+1\right) \sum_{j=0}^{p-2} \chi\left(-p^{-1} e_{1}^{N+1-j\left(p^{2}+p+1\right)}\right)+1
\end{aligned}
$$

Обозначим через $\Sigma$ выражение в левой части (2.24). Поскольку $K$ - неразветвленное расширение $Q_{p}$, можно получить характер ранга нуль, полагая

$$
\chi(x)=\chi_{p}(\operatorname{Tr}(x))
$$

Класс элемента $\operatorname{Tr}(x), x \in O$, в $\mathbb{F}_{p}$ совпадает с $\operatorname{tr}(\hat{x})$, где $\hat{x}-$ класс $x$ в $\mathbb{F}_{q}$ (см. [15]). Выражение $\chi_{p}\left(-p^{-1} \sigma\right), \sigma \in \mathbb{Z}_{p}$, определяет нетривиальный аддитивный характер $X$ на конечном поле $\mathbb{F}_{p}$. Пусть $\delta=\lambda_{1}^{p^{2}+p+1}$. Элементы $0,1, \delta, \delta^{2}, \ldots, \delta^{p-2}$ образуют подполе в $\mathbb{F}_{q}$, совпадающее с $\mathbb{F}_{p}$. Следовательно,

$$
\chi\left(-p^{-1} e_{1}^{N+1-j\left(p^{2}+p+1\right)}\right)=X\left(\delta^{-j} \operatorname{tr}\left(\lambda_{1}^{N+1}\right)\right)
$$

откуда

$$
\Sigma=\left\{\begin{array}{lll}
p^{3}, & \text { если } & \operatorname{tr}\left(\lambda_{1}^{N+1}\right)=0, \\
-p^{2}-p, & \text { если } & \operatorname{tr}\left(\lambda_{1}^{N+1}\right) \neq 0 .
\end{array}\right.
$$

Подставляя это в (2.23), находим

$$
E(x)=\|x\|^{2 \alpha / 3-1} r(x)
$$

где

$$
r(x)=\left(1+\frac{p^{\alpha}-1}{p^{2}+p+1}\right) \frac{1-p^{-2 \alpha}}{1-p^{2 \alpha-3}}+\frac{p^{3-2 \alpha}\left(p^{\alpha}-1\right)}{p^{2}+p+1},
$$

если $\operatorname{tr}\left(\lambda_{1}^{N+1}\right)=0$, или

$$
r(x)=\left(1+\frac{p^{\alpha}-1}{p^{2}+p+1}\right) \frac{1-p^{-2 \alpha}}{1-p^{2 \alpha-3}}-\frac{p^{1-2 \alpha}\left(p^{\alpha}-1\right)(p+1)}{p^{2}+p+1}
$$


если $\operatorname{tr}\left(\lambda_{1}^{N+1}\right) \neq 0$.

Аналогично при $\alpha=3 / 2$ имеем

$$
\begin{aligned}
& E(x)=\frac{(p+2)(1-p)}{3 p^{2} \log p} \log \|x\|+\frac{p^{-3}(p-1)}{p^{2}+p+1} r_{1}(x), \\
& r_{1}(x)=\left\{\begin{array}{lll}
p^{3}, & \text { если } & \operatorname{tr}\left(\lambda_{1}^{N+1}\right)=0, \\
-p(p+1), & \text { если } & \operatorname{tr}\left(\lambda_{1}^{N+1}\right) \neq 0 .
\end{array}\right.
\end{aligned}
$$

В обоих случаях подобные рассуждения дают также следуюшее выражение для $\Omega$ :

$$
\Omega(u)=\left(1+\frac{p^{-\alpha}-1}{p^{2}+p+1}\right) \frac{1-p^{2 \alpha}}{1-p^{-2 \alpha-3}}+\frac{p^{2 \alpha+3}\left(p^{-\alpha}-1\right)}{p^{2}+p+1},
$$

если $\operatorname{tr}\left(\lambda_{1}^{N+1}\right)=0$, или

$$
\Omega(u)=\left(1+\frac{p^{-\alpha}-1}{p^{2}+p+1}\right) \frac{1-p^{2 \alpha}}{1-p^{-2 \alpha-3}}-\frac{p^{2 \alpha+1}\left(p^{-\alpha}-1\right)(p+1)}{p^{2}+p+1},
$$

если $\operatorname{tr}\left(\lambda_{1}^{N+1}\right) \neq 0$. Как и раньше, $N$ связано с $u \in U$ формулой (2.22).

Формулы $(2.21),(2.25),(2.26)$ для фундаментального решения станут яснее, если выразить условие $\operatorname{tr}\left(\lambda_{1}^{N+1}\right)=0$ на $x \in K, x=x_{1} e_{1}+x_{2} e_{2}+x_{3} e_{3}, x_{j} \in Q_{p}$, в терминах коэффициентов $x_{j}$.

ПРЕДЛОЖенИЕ 2.3. Пусть $u_{x}=e_{1}^{N} y,\|1-y\|<1$. Равенство $\operatorname{tr}\left(\lambda_{1}^{N+1}\right)=0$ әквивалентно неравенству

$$
\left|x_{1}\right|_{p}<\max \left\{\left|x_{2}\right|_{p},\left|x_{3}\right|_{p}\right\} .
$$

ДокАЗАТЕЛЬСТВо. Если $N=p$ или $N=p^{2}, \operatorname{to~} \operatorname{tr}\left(\lambda_{1}^{N+1}\right)=0$, поскольку базис $\left\{\lambda_{1}, \lambda_{2}, \lambda_{3}\right\}$ автодуальный, $\lambda_{2}=\lambda_{1}^{p}, \lambda_{3}=\lambda_{1}^{p^{2}}$. Рассмотрим множество $\mathfrak{N}=\operatorname{ker} \operatorname{tr} \subset \mathbb{F}_{q}$, являюшееся векторным подпространством в $\mathbb{F}_{q}$ над $\mathbb{F}_{p}$. Очевидно, $\operatorname{dim} \mathfrak{N} \leqslant 2$. С другой стороны, векторы $\lambda_{1}^{p+1} \in \mathfrak{N}_{\text {и }} \lambda_{1}^{p^{2}+1} \in \mathfrak{N}$ линейно независимы над $\mathbb{F}_{p}$, иначе было бы $\lambda_{1}^{p^{2}-p} \in \mathbb{F}_{p}$, что противоречит описанию $\mathbb{F}_{p} \backslash\{0\}$ как множества элементов $\lambda_{1}^{\nu\left(p^{2}+p+1\right)}, \nu=0,1, \ldots, p-2$.

Следовательно, множество $\left\{x \in K: \operatorname{tr}\left(\lambda_{1}^{N+1}\right)=0\right\}$ состоит из тех $x$, для котоpыx

$$
\lambda_{1}^{N+1}=\sigma_{1} \lambda_{1} \lambda_{2}+\sigma_{2} \lambda_{1} \lambda_{3}, \quad \sigma_{1}, \sigma_{2} \in \mathbb{F}_{p} .
$$

Это эквивалентно сравнению

$$
u_{x} \equiv s_{2} e_{2}+s_{3} e_{3} \quad(\bmod P),
$$

где $s_{2}, s_{3} \in Q_{p}, \max \left\{\left|s_{2}\right|_{p},\left|s_{3}\right|_{p}\right\}=1$. Отсюда

$$
x=p^{-m}\left(s_{2} e_{2}+s_{3} e_{3}\right)+x^{\prime}, \quad\|x\|=q^{m}, \quad\left\|x^{\prime}\right\|<q^{m}
$$

(для неразветвленного расширения $\beta=p$ ). Это равенство означает, что коэффициенты разложения $x=x_{1} e_{1}+x_{2} e_{2}+x_{3} e_{3}$ удовлетворяют неравенству (2.27). Предложение доказано. 
2.6. Кватернарные формы. Известно (см. [7]), что квадратичная форма $h\left(\xi_{1}, \xi_{2}, \xi_{3}, \xi_{4}\right)$, удовлетворяюшая условию эллиптичности $(2.15)$, единственна с точностью до линейного изоморфизма. Такая форма представима в виде

$$
h\left(\xi_{1}, \xi_{2}, \xi_{3}, \xi_{4}\right)=\xi_{1}^{2}-s \xi_{2}^{2}-\sigma \xi_{3}^{2}+s \sigma \xi_{4}^{2},
$$

где $s, \sigma \in Q_{p}$, и символ Гильберта $(s, \sigma)=-1$. В частности, можно взять $\sigma=p$, $s \in \mathbb{Z}-$ квадратичный невычет $\bmod p$.

Пусть $K=\left(\frac{s, p}{Q_{p}}\right)$ - тело кватернионов над $Q_{p}$ с базисом $1, i, j, k$ и таблицей умножения

$$
\begin{gathered}
i^{2}=s, \quad j^{2}=p, \quad i j=-j i=k, \quad k^{2}=-s p, \\
i k=-k i=j s, \quad j k=-k j=-i p .
\end{gathered}
$$

$K$ является центральной алгеброй с делением над $Q_{p}$ и с нормализованной абсолютной величиной

$$
\|x\|=\left|h\left(x_{1}, x_{2}, x_{3}, x_{4}\right)\right|_{p}^{2}, \quad x=x_{1}+x_{2} i+x_{3} j+x_{4} k .
$$

$\mathrm{B}$ данном случае $q=p^{2}, \beta=j$. Приведенный след $t: K \rightarrow Q_{p}$ равен $t(x)=2 x_{1}$.

Базис координатного представления был найден в [8]:

$$
e_{1}=\frac{1}{2}(1-j), \quad e_{2}=\frac{1}{2}(1+j), \quad e_{3}=\frac{1}{2 s}(i-k), \quad e_{4}=\frac{1}{2}(i+k) .
$$

Согласно [8] $\mu_{1}=-1, \mu_{2}=1, \mu_{3}=-s^{-1}, \mu_{4}=s, c=1$. Полагая $\mathfrak{A}=D_{K}^{\alpha / 2}$,

$$
T=\left(\begin{array}{cccc}
-1 & 0 & 1 & 0 \\
1 & 0 & 1 & 0 \\
0 & -1 & 0 & 1 \\
0 & s & 0 & s
\end{array}\right), \quad|\operatorname{det} T|_{p}=1
$$

(см. [8]), получаем гиперсингулярное интегральное представление оператора $A$ с $\Omega(u) \equiv \frac{1-p^{\alpha}}{1-p^{-\alpha-2}}$. Фундаментальное решение записывается в виде

$$
\begin{gathered}
G\left(x_{1}, x_{2}, x_{3}, x_{4}\right)=E\left(\frac{x_{3}-x_{1}}{2} e_{1}+\frac{x_{1}+x_{3}}{2} e_{2}+\frac{x_{4}-x_{2}}{2} e_{3}+\frac{x_{2}+x_{4}}{2} e_{4}\right), \\
E(x)=\left\{\begin{array}{l}
\frac{1-p^{-\alpha}}{1-p^{\alpha-2}}\|x\|^{\alpha / 2-1}, \\
\frac{1-p^{2}}{2 p^{2} \log p} \log \|x\|, \quad \text { если } \alpha \neq 2,
\end{array}\right.
\end{gathered}
$$

Заметим, что функция Грина, изучавшаяся в [8], фактически является фундаментальньм решением, связанным с псевдодифференциальным оператором с символом $|h(\xi)|^{\alpha}+\lambda, \lambda>0$. Пользуясь случаем, отметим опечатку в [8], относящуюся к случаю кватернарных форм: коэффициент в асимптотике функции Грина (последняя формула в [8]) должен быть равен $\frac{p^{\alpha+2}\left(p^{\alpha}-1\right)}{p^{\alpha+2}-1} \lambda^{-2}$.

Поскольку квадратичных форм с $n \geqslant 5$, удовлетворяющих (2.15), не существует [6], [7], приведенные результаты завершают доказательство теоремы 2.1 . 


\section{§ 3. Гиперболические уравнения}

3.1. Уравнения с одной пространственной переменной. Рассмотрим уравнение $A z=f$, где $A$ - псевдодифференциальный оператор (1.5) с $n=2$, $h(\xi)=\xi_{1}^{2}-\xi_{2}^{2}, \alpha>0$. Как и в случае вешественного волнового уравнения, этот оператор можно преобразовать (очевидной заменой переменных) в произведение двух одномерных операторов типа $D^{\alpha}$. Получаются гиперсингулярное интегральное представление

$$
\begin{aligned}
(A u)(x, t)= & \left(\frac{1-p^{\alpha}}{1-p^{-\alpha-1}}\right)^{2} \int_{Q_{p}^{2}}|y \theta|_{p}^{-\alpha-1}[u(x-y-\theta, t-y+\theta)- \\
& -u(x-\theta, t+\theta)-u(x-y, t-y)+u(x, t)] d y d \theta
\end{aligned}
$$

и формулы для фундаментального решения (понимаемого так же, как в эллиптическом случае)

$$
G(x, t)=\left(\frac{1-p^{-\alpha}}{1-p^{\alpha-1}}\right)^{2}|x+t|_{p}^{\alpha-1}|x-t|_{p}^{\alpha-1}
$$

если $\alpha \neq 1$, или

$$
G(x, t)=\left(\frac{p-1}{p \log p}\right)^{2}\left(\log |x+t|_{p}\right) \log |x-t|_{p}
$$

если $\alpha=1$.

3.2. Уравнения с несколькими пространственными переменными. Рассмотрим уравнение $A z=f$, где $A$-псевдодифференциальный оператор (1.5) с $h\left(\xi_{1}, \ldots, \xi_{n}\right)=\xi_{1}^{2}-g\left(\xi_{2}, \ldots, \xi_{n}\right)$,

$$
g\left(\xi_{2}, \ldots, \xi_{n}\right) \neq 0, \text { если }\left(\xi_{2}, \ldots, \xi_{n}\right) \neq 0 .
$$

Обозначим через $P_{\alpha}$ символ $\left|h\left(\xi_{1}, \ldots, \xi_{n}\right)\right|_{p}^{\alpha}$. Квадратичные формы $g$, удовлетворяюшие (3.1), существуют только при $n \leqslant 5$. Ниже предполагается, что $n \geqslant 3$. Посредством замены переменных можно представить $g$ как сумму квадратов с ненулевыми коэффициентами из $\mathbb{Z}_{p}$.

Функциональное пространство $\mathscr{D}_{C}\left(Q_{p}^{n}\right)$ (см. введение) является замкнутым подпространством в $\mathscr{D}\left(Q_{p}^{n}\right)$. Преобразование Фурье, вообше говоря, выводит из $\mathscr{D}_{C}\left(Q_{p}^{n}\right)$. Однако для оператора $A=F^{-1} P_{\alpha} F$ имеет место обратное.

ПРЕДЛОЖенИЕ 3.1. Оператор А непрерывно действует в $\mathscr{D}_{C}\left(Q_{p}^{n}\right)$. 
ДокАЗАТЕЛЬСТво. Пусть $\varphi \in \mathscr{D}_{C}\left(Q_{p}^{n}\right)$. Тогда $\widetilde{\varphi} \in \mathscr{D}\left(Q_{p}^{n}\right)$. Предположим, что

$$
\begin{gathered}
\operatorname{supp} \widetilde{\varphi} \subset\left\{\xi \in Q_{p}^{n}: \max _{1 \leqslant j \leqslant n}\left|\xi_{j}\right|_{p} \leqslant p^{N}\right\}, \\
\widetilde{\varphi}\left(\xi+\xi^{\prime}\right)=\widetilde{\varphi}(\xi), \text { если } \max _{1 \leqslant j \leqslant n}\left|\xi_{j}^{\prime}\right|_{p} \leqslant p^{l},
\end{gathered}
$$

$N, l \in \mathbb{Z}$. Можно считать, что $N \geqslant 0, l \leqslant N-1$.

Докажем существование такого числа $\nu \in \mathbb{Z}$, зависяшего только от $N$ и $l$, что

$$
\widetilde{\varphi}\left(\xi_{1}, \ldots, \xi_{n}\right)=0, \text { если }\left|h\left(\xi_{1}, \ldots, \xi_{n}\right)\right|_{p}<p^{\nu} .
$$

Достаточно рассмотреть $\xi_{1}, \ldots, \xi_{n} \mathrm{c} \max _{1 \leqslant j \leqslant n}\left|\xi_{j}\right|_{p} \leqslant p^{N}$. Обозначим $\eta_{j}=$ $p^{N} \xi_{j}, j=1, \ldots, n$. Тогда $\eta_{j} \in \mathbb{Z}_{p}$. Если $\left|h\left(\xi_{1}, \ldots, \xi_{n}\right)\right|_{p}<p^{\nu}$, то

$$
\left|h\left(\eta_{1}, \ldots, \eta_{n}\right)\right|_{p}<p^{\nu+2 N} .
$$

Имеются две возможности: либо

$$
\max _{1 \leqslant j \leqslant n}\left|\eta_{j}\right|_{p} \leqslant p^{l-N}
$$

так что $\max _{1 \leqslant j \leqslant n}\left|\xi_{j}\right|_{p} \leqslant p^{l}$ и $\widetilde{\varphi}\left(\xi_{1}, \ldots, \xi_{n}\right)=\widetilde{\varphi}(0, \ldots, 0)=0$, либо хотя бы для одного значения $j\left|\eta_{j}\right|_{p} \geqslant p^{l-N+1}$. Пусть, например, $\left|\eta_{1}\right|_{p}=p^{k}, l-N+1 \leqslant k \leqslant 0$.

При $\nu<2 k-2 N$ можно использовать вариант леммы Гензеля, доказанный Серром $[7$, гл. 2 , теорема 1$]$. Согласно этому результату существуют такие $\eta_{j}^{0} \in$ $\mathbb{Z}_{p}, j=1, \ldots, n$, что

$$
h\left(\eta_{1}^{0}, \ldots, \eta_{n}^{0}\right)=0, \quad \max \left|\eta_{j}^{0}-\eta_{j}\right|_{p} \leqslant p^{-k+\nu+2 N} .
$$

Пусть $\xi_{j}^{0}=p^{-N} \eta_{j}^{0}, j=1, \ldots, n$. Тогда $\left(\xi_{1}^{0}, \ldots, \xi_{n}^{0}\right) \in C$,

$$
\max _{1 \leqslant j \leqslant n}\left|\xi_{j}-\xi_{j}^{0}\right|_{p} \leqslant p^{N+\nu-k} \leqslant p^{2 N+\nu-l-1} .
$$

Если выбрать $\nu \leqslant 2 l-4 N+1$, то $\nu<2 k-2 N$ (условие применимости предыдущей конструкции),

$$
\max _{1 \leqslant j \leqslant n}\left|\xi_{j}-\xi_{j}^{0}\right|_{p} \leqslant p^{l}
$$

откуда $\widetilde{\varphi}\left(\xi_{1}, \ldots, \xi_{n}\right)=0$.

Функция $P_{\alpha}$ локально постоянна в окрестности носителя $\widetilde{\varphi}$. Отсюда следует включение $P_{\alpha} \widetilde{\varphi} \in \mathscr{D}\left(Q_{p}^{n}\right)$. Таким образом, $A \varphi \in \mathscr{D}_{C}\left(Q_{p}^{n}\right)$. Непрерывность $A$ вытекает из определения топологии в $\mathscr{D}_{C}\left(Q_{p}^{n}\right)$, индуцированной из $\mathscr{D}\left(Q_{p}^{n}\right)$. Предложение доказано. 
Сопряженный оператор $A^{\prime}: \mathscr{D}_{C}^{\prime}\left(Q_{p}^{n}\right) \rightarrow \mathscr{D}_{C}^{\prime}\left(Q_{p}^{n}\right)$ является расширением оператора $A$. Заметим, что преобразование $Ф$ урье не определено на $\mathscr{D}_{C}^{\prime}\left(Q_{p}^{n}\right)$, так как оно не сохраняет $\mathscr{D}_{C}\left(Q_{p}^{n}\right)$.

Свертка основных функций и обрашение $\check{\varphi}(x)=\varphi(-x)$ корректно определены в $\mathscr{D}_{C}\left(Q_{p}^{n}\right)$. Стандартным образом определяется свертка обобщенной функции с основной. Ниже фундаментальное решение будет пониматься как решение $E \in$ $\mathscr{D}_{C}^{\prime}\left(Q_{p}^{n}\right)$ уравнения $A^{\prime} E=\delta$. Если $E$ - фундаментальное решение, $f \in \mathscr{D}_{C}\left(Q_{p}^{n}\right)-$ основная функция, то функция $z=E * f$, рассматриваемая как обобшенная функция из $\mathscr{D}_{C}^{\prime}\left(Q_{p}^{n}\right)$, удовлетворяет уравнению $A^{\prime} z=f$.

Для построения фундаментального решения $E \in \mathscr{D}_{C}^{\prime}\left(Q_{p}^{n}\right)$ достаточно рассмотреть функцию

$$
P_{-\alpha}\left(\xi_{1}, \ldots, \xi_{n}\right)=\left|h\left(\xi_{1}, \ldots, \xi_{n}\right)\right|_{p}^{-\alpha}
$$

как обобшенную функцию из $\mathscr{D}^{\prime}\left(Q_{p}^{n} \backslash C\right)$ и положить

$$
E=F P_{-\alpha},
$$

где преобразование $Ф$ урье, действующее как изоморфизм из $\mathscr{D}_{C}\left(Q_{p}^{n}\right)$ на $\mathscr{D}\left(Q_{p}^{n} \backslash C\right)$, продолжено до действия $\mathscr{D}^{\prime}\left(Q_{p}^{n} \backslash C\right) \rightarrow \mathscr{D}_{C}^{\prime}\left(Q_{p}^{n}\right)$ (отметим, что функции $h$ и $P_{\lambda}$ четные, так что здесь можно не различать прямые и обратные преобразования).

Известно [27], [28], что функция $P_{\lambda}, \lambda \in(0, \infty)$, со значениями в $\mathscr{D}^{\prime}\left(Q_{p}^{n}\right)$ допускает аналитическое продолжение до мероморфной функции, определенной на всей комплексной плоскости, с простыми полюсами в точках $-1+\frac{2 k \pi i}{\log p},-\frac{n}{2}+\frac{k \pi i}{\log p}$ $(k \in \mathbb{Z})$. Из сказанного вытекает следуюший результат.

Теорема 3.1. Пусть $\alpha \neq 1$ u $\alpha \neq n / 2$. Тогда фундаментальное решение (3.2) допускает продолжение до обобщенной функции из $\mathscr{D}^{\prime}\left(Q_{p}^{n}\right)$.

\section{Список литературы}

1. Владимиров В. С., Волович И. В., Зеленов Е. И. p-адический анализ и математическая физика. М.: Наука, 1994.

2. Khrennikov A. Yu. p-adic valued distributions in mathematical physics. Dordrecht: Kluwer, 1994.

3. Ruelle P., Thiran E., Verstegen D., Weyers J. Quantum mechanics on $p$-adic fields // J. Math. Phys. 1989. V. 30. № 12. P. 2854-2874.

4. Владимиров В. С. Обобщенные функции над полем $p$-адических чисел // УМН. 1988. T. 43. № 5. C. 17-53.

5. Haran S. Quantization and symbolic calculus over the $p$-adic numbers // Ann. Inst. Fourier. 1993. V. 43. № 4. P. 997-1053.

6. Боревич З. И., Шафаревич И. Р. Теория чисел. М.: Наука, 1985.

7. Серр Ж.-П. Курс арифметики. М.: Мир, 1972.

8. Кочубей А. Н. О p-адических функциях Грина // ТМФ. 1993. Т. 96. №1. С. 123-139.

9. Vladimirov V.S., Volovich I. V. p-adic quantum mechanics // Comm. Math. Phys. 1989. V. 123. № 4. P. 659-676.

10. Хренников А. Ю. Фундаментальные решения над полем $p$-адических чисел // Алгебра и анализ. 1992. Т. 4. № 3. С. 248-266.

11. Kochubei A. N. A Schrödinger type equation over the field of $p$-adic numbers // J. Math. Phys. 1993. V. 34. № 8. P. 3420-3428. 
12. Кочубей А.Н. Параболические уравнения над полем $p$-адических чисел // Изв. АН CCCP. 1991. T. 55. №6. C. 1312-1330.

13. Алгебраическая теория чисел / Ред. Дж. Касселс и А. Фрёлих. М.: Мир, 1969.

14. Гельфанд И.М., Граев М.И., Пятецкий-Шапиро И.И. Теория представлений и автоморфные функции. М.: Наука, 1966.

15. Вейль А. Основы теории чисел. М.: Мир, 1972.

16. Sally P.J., Taibleson M. H. Special functions on locally compact fields // Acta Math. 1966. V. 116. № 3-4. P. 279-309.

17. Бурбаки Н. Коммутативная алгебра. М.: Мир, 1971.

18. Пирс Р. Ассоциативные алгебры. М.: Мир, 1986.

19. Saloff-Coste L. Opératéurs pseudo-différentiels sur certains groupes totalement discontinus // Studia Math. 1986. V. 83. № 3. P. 205-228.

20. Артин Э. Геометрическая алгебра. М.: Наука, 1969.

21. Narkiewicz W. Elementary and analytic theory of algebraic numbers. Warszawa: PWN, 1974.

22. Лидл Р., Нидеррайтер Г. Конечные поля. М.: Мир, 1988.

23. Taibleson $M$. $H$. The existence of natural field structures for finite dimensional vector spaces over local fields // Pacif. J. Math. 1976. V. 63. № 2. P. 545-551.

24. Lempel A., Weinberger M. J. Self-complementary normal bases in finite fields // SIAM J. Discr. Math. 1988. V. 1. P. 193-198.

25. Davenport H. Bases for finite fields // J. London Math. Soc. 1968. V. 43. № 1. P. 21-39.

26. Lempel $A$. Characterization and synthesis of self-complementary normal bases in finite fields // Linear Algebra Appl. 1988. V. 98. P. 331-346.

27. Ono T. Gauss transform and zeta-functions // Ann. Math. 1970. V. 91. № 2. P. 332-361.

28. Граев М.И., Прохорова Р. И. Однородные обобщенные функции в векторном пространстве над локальным неархимедовым полем, связанные с квадратичной формой // Функц. анализ и его прилож. 1972. Т. 6. № 3. С. 70-71.

Институт математики

Поступило в редакцию

Национальной академии наук Украины

25.IV.1997 